\title{
Newly developed post-operative atrial fibrillation is associated with an increased risk of late recurrence of atrial fibrillation in patients who underwent open heart surgery: Long-term follow up
}

\author{
Yae Min Park ${ }^{1}$, Mi Suk Cha ${ }^{1}$, Chul-Hyun Park ${ }^{2}$, Chang Hu Choi ${ }^{2}$, \\ Yang Bin Jeon ${ }^{2}$, Woong Chol Kang ${ }^{1}$, In Suck Choi ${ }^{1}$, Kook Yang Park ${ }^{2}$ \\ ${ }^{1}$ Cardiology Division, Gachon University Gil Medical Center, Incheon, Korea
}

${ }^{2}$ Department of Thoracic and Cardiovascular Surgery, Gachon University Gil Medical Center, Incheon, Korea

\begin{abstract}
Background: Herein is sought to determine whether the occurrence of post-operative atrial fibrillation $(P O A F)$ increases the risk of late recurrence of atrial fibrillation $(A F)$ in patients undergoing open heart surgery (OHS).

Methods: This study included 938 patients (56.7 \pm 13.1 years old, 550 males) with no history of $A F$ who underwent OHS. All patients were monitored continuously for development of POAF after surgery until the time of hospital discharge and received clinical follow up with serial evaluation of rhythm status.

Results: Among the total population, POAF occurred in 207 (22.1\%) patients and late AF in 88 (9.4\%) patients during the mean follow up period of $78.1 \pm 39.1$ months. Development of late AF occurred more frequently in patients with POAF than in those without [29.0\% (60/207) vs. 3.8\% (28/731), $p<0.01$ ]. Higher septal E/e' ratio (HR 1.04, 95\% CI 1.00-1.08, $p=0.04$ ) was an independent predictor of late occurrence of $A F$ and an episode of POAF (HR 27.12, 95\% CI 8.46-86.96, $p<0.01$ ) was the most powerful predictor.

Conclusions: $P O A F$ is significantly associated with an increased risk of late AF recurrence during long-term follow up. Careful concern regarding late recurrence of $A F$ with serial evaluation of rhythm status is required in patients with POAF. (Cardiol J 2017; 24, 6: 633-641)
\end{abstract}

Key words: post-operative atrial fibrillation, open heart surgery, late recurrence

\section{Introduction}

Post-operative atrial fibrillation (POAF) is a frequent complication affecting $20-40 \%$ of patients following open heart surgery (OHS). POAF after cardiac surgery is usually self-limiting [1], and most patients are in sinus rhythm at discharge. However, POAF can sometimes result in increased risk of post-operative stroke, morbidity, and prolonged hospitalization [2-4]. Recent studies have suggested that POAF following coronary artery bypass surgery (CABG) carries a risk of future atrial fibrillation (AF) recurrence and higher mortality than patients without an episode of POAF $[5,6]$. However, late occurrence rate of AF during long-term follow up in patients receiving overall OHS has yet to be determined.

This study investigated the relationships between POAF and late occurrence of AF during long-term follow up in a large cohort of patients

Address for correspondence: Chul-Hyun Park, MD, Department of Thoracic and Cardiovascular Surgery, Gachon University Gil Hospital, 774-21 Namdong Daero, Namdonggu, Incheon, Republic of Korea, 21556, tel: 82-32-460-3663,

fax: 82-32-469-1906, e-mail: ypruimin@gmail.com; cdgpch@gilhospital.com

Received: 14.03 .2017

Accepted: 12.05.2017 
who underwent OHS. It was hypothesized that $\mathrm{POAF}$ is a risk factor for late occurrence of $\mathrm{AF}$.

\section{Methods}

\section{Patients}

This retrospective study included 938 adult patients $(56.7 \pm 13.1$ years old, 550 males $)$ with no prior history of AF among 1483 patients who underwent OHS between January 1999 and June 2010. Exclusion criteria were: 1 ) patients who had previously documented AF before surgery $(n=260)$, 2) patients with another form of significant arrhythmia which might affect the occurrence of AF such as sinus node dysfunction $(\mathrm{n}=5), 3)$ patients who had undergone permanent pacemaker implantation prior to surgery $(\mathrm{n}=5), 4)$ patients who had undergone OHS previously $(\mathrm{n}=73)$ and 4) patients who did not receive clinical follow up for more than 1 year $(n=202)$. All patients were followed up regularly in the outpatient clinic. Finally, 938 patients were enrolled in this study and were divided into two groups based on the development of POAF (POAF group; $\mathrm{n}=207$, and no POAF group; $\mathrm{n}=731$ ).

Clinical characteristics including demographics, cardiovascular risk factors, laboratory data, peri/post-operative information, and clinical outcomes were obtained through chart review. Hypertension was defined as systolic blood pressure $\geq 140 \mathrm{~mm} \mathrm{Hg}$ or diastolic blood pressure $\geq 90 \mathrm{~mm} \mathrm{Hg}$, or if they were already being treated for this condition. Diabetes mellitus was defined as fasting glucose $\geq 126 \mathrm{mg} / \mathrm{dL}$ or $2 \mathrm{~h}$ post-prandial glucose $\geq 200 \mathrm{mg} / \mathrm{dL}$ or glycated hemoglobin (HbA1c) $\geq 6.5 \%$, or if they were already being treated for this condition. Renal dysfunction was defined as serum creatinine elevation above $1.2 \mathrm{mg} / \mathrm{dL}$ with or without the need for hemodialysis. The elevation of creatinine value was confirmed with more than one determination before surgery. For diagnosis of dyslipidemia, triglyceride $(\geq 150 \mathrm{mg} / \mathrm{dL})$ and high density lipoprotein-cholesterol (HDL-C) levels $(<40 \mathrm{mg} / \mathrm{dL}$ for males and $<50 \mathrm{mg} / \mathrm{dL}$ for females $)$ were used as parameters [7]. High sensitivity $\mathrm{C}$-reactive protein (hsCRP) levels were determined using a turbidimetic assay (Denka Seiken, Tokyo, Japan) using the Hitachi 7600-110. Laboratory tests were repeated within $48 \mathrm{~h}$ after the operation. Diagnosis of post-operative infection was established if at least one of the following criteria were present: 1) positive blood, urine or surgical wound discharge cultures, 2) pyrexia $>38^{\circ} \mathrm{C}$ for at least 2 days beyond the first 2 post-operative days, associated with white cell count elevation, 3) radiologic indication of significant lung consolidation, and 4) clinical signs of significant surgical wound infection [8].

The local Institutional Review Board approved this study and all patients provided written informed consent.

\section{Evaluation of rhythm status}

All patients were treated with beta-blocker at least 7 days before the operation, unless contraindicated. Prophylactic anti-arrhythmic drugs were not administered. Patients were continuously monitored by bedside electrocardiogram (ECG) monitoring of lead II for the first $48 \mathrm{~h}$ after surgery. After transferring to general wards, the arrhythmias were detected by regular 4-h clinical assessment and a standard 12-lead ECG which was routinely performed every day until discharge. Continuous ECG monitoring or Holter monitoring was reinstituted if patients complained of symptoms suggesting arrhythmia during hospitalization. POAF was defined as a newly developed AF episode lasting more than 1 min documented by 12-lead ECG or continuous monitoring during the first 1 month after surgery and late occurrence of $\mathrm{AF}$ was defined as occurrence of AF after 1 month. Onset, duration of $\mathrm{AF}$, management of $\mathrm{AF}$, and rhythm status at discharge were reviewed in the patient's record.

\section{Echocardiographic measurements}

Echocardiographic measurements were obtained before the operation in all patients. The 2-dimensional and M-mode echocardiograms were obtained according to the recommendation of the American Society of Echocardiography, with patients in the left lateral decubitus position [9]. Left atrial (LA) dimensions were measured using the parasternal long-axis and apical 4-chamber views at ventricular end systole (maximum LA size). The left ventricular (LV) end-diastolic and end-systolic dimensions were measured using M-mode echocardiography recorded from the para-sternal long axis view. The LV end-diastolic, end-systolic volumes and ejection fraction (EF) were measured by the modified bi-plane Simpson rule [10]. Diastolic function was determined based on Doppler echocardiography and evaluated by the analysis of peak trans-mitral inflow velocities (E: early diastolic mitral flow velocity, A: late diastolic mitral flow velocity). The deceleration time of the $\mathrm{E}$ waves was also measured. Peak velocities during early (E') and late (A') diastole at the level 
of the septal mitral annulus were measured using pulsed-wave tissue Doppler. Thereafter, E/A and $\mathrm{E} / \mathrm{e}$ ' ratios reflecting $\mathrm{LV}$ end-diastolic pressure were calculated. At least three cardiac cycles were averaged for each parameter measurement.

\section{Type of OHS}

The type of surgery was classified as isolated CABG, valve surgery, aorta surgery, or combined surgery. Off-pump coronary bypass surgery (OPCAB) was performed in selected patients at the discretion of the physician. Heart transplantation $(\mathrm{n}=4)$, mass excision $(\mathrm{n}=20)$, and surgery for congenital heart disease $(n=57)$ were classified as miscellaneous.

\section{Management of POAF}

All patients with POAF were managed with rate control drugs including beta-blockers, unless contraindicated, and anticoagulation was administered in patients with continuous AF more than $48 \mathrm{~h}$ after the onset of POAF. Highly symptomatic patients or patients with failure of medications in controlling the ventricular rate led to cardioversion using anti-arrhythmic drugs or by electrical cardioversion at the suggestion of a previous study stating that a primary rate-control strategy may be equivalent to a strategy that restores sinus rhythm [11]. Patients who were discharged to their homes in AF rhythm were maintained on warfarin (in the absence of any contra-indication) and were usually referred for cardioversion after 3-6 weeks. In the absence of evidence of AF recurrence 3 months subsequent to the operation, anti-arrhythmic drugs were usually stopped at the discretion of the physician. If patients remained in $\mathrm{AF}$, other medications were added or cardioversion was considered.

\section{Clinical follow up}

The patients were seen in an outpatient clinic at 1 week and then $1,3,6,9$, and 12 months after discharge, subsequently every 3 or 6 months thereafter. A 12-lead surface ECG was performed at every visit. A detailed history of the patients' symptoms suggesting potential for arrhythmia occurrence was taken and 24-h or 48-h Holter monitoring was performed in those patients. All patients received clinical follow up with serial evaluation of rhythm status for more than 1 year.

\section{Statistical analysis}

Patients were classified according to two groups and those who did not develop POAF served as the control group. Continuous variables are reported as mean \pm standard deviation, while categorical variables are reported as a number or percentage. Student t test was used for comparison of continuous variables, while categorical variables were compared using $\chi^{2}$ test or the Fisher exact test, as appropriate among groups. AF free survival (any occurrence of atrial arrhythmia) was estimated using the Kaplan-Meier method and AF free survival was compared between POAF group and without POAF group using a Wilcoxon log rank test. Multivariate analysis was performed for analysis of the independent predictors of late occurrence of AF. Univariate variables with $\mathrm{p}<0.05$ were entered into the model (control for confounding). All tests were 2 -tailed and a p-value of $<0.05$ was considered significant. All statistical analyses were performed using SPSS 12.0 software (SPSS, Inc., an IBM Company, Chicago, Illinois, USA).

\section{Results}

\section{Baseline characteristics according to the POAF}

Among the total population, 207 (22.1\%) patients developed POAF. Baseline characteristics according to development of POAF are compared in Table 1. Patients with POAF were significantly older than those without $(60.4 \pm 11.2$ vs. $55.7 \pm$ \pm 13.5 year, $\mathrm{p}<0.01)$. Patients with POAF showed significantly higher hsCRP level $(11.8 \pm 6.2$ vs. $10.3 \pm 7.2 \mathrm{mg} / \mathrm{dL}, \mathrm{p}=0.04)$ after the operation. $\mathrm{LA}$ diameter $(42.4 \pm 8.4$ vs. $40.7 \pm 6.7 \mathrm{~mm}, \mathrm{p}=0.02)$ and LA volume $(71.4 \pm 39.0$ vs. $63.0 \pm 33.3 \mathrm{~mL}$, $\mathrm{p}=0.04$ ) were significantly larger in patients with POAF than those without. E/e' was significantly higher in patients with POAF $(16.9 \pm 11.8$ vs. $14.7 \pm 7.8, \mathrm{p}=0.04)$. Other echocardiographic measurements including LVEF were similar between the two groups. The incidence of POAF differed significantly according to the type of surgery, with the highest frequency in patients undergoing combined aorta and valvular surgery $(34.4 \%)$ followed by isolated valvular surgery $(33.0 \%)$. The POAF group included significantly fewer patients who underwent $\mathrm{OPCAB}$ than the no POAF group (19.3\% [40/207] vs. $32.3 \%$ [236/731], p < 0.01). A significantly higher proportion of patients with POAF underwent mitral valvular surgery $(21.2 \%$ [44/207] vs. $10.3 \%$ [75/731], p < 0.01). Post-operative infection rate was similar between the two groups. Otherwise, clinical characteristics and the presence of co-morbidities were similar between the two groups. 
Table 1. Patient characteristics according to development of post-operative atrial fibrillation (POAF).

\begin{tabular}{|c|c|c|c|}
\hline & $\operatorname{POAF}(+) ; n=207$ & $\operatorname{POAF}(-) ; n=731$ & $\mathbf{P}$ \\
\hline Age [years] & $60.4 \pm 11.2$ & $55.7 \pm 13.5$ & $<0.01 \ddagger$ \\
\hline Male & $114(55.1 \%)$ & $436(59.6 \%)$ & 0.24 \\
\hline Hypertension & $120(58.0 \%)$ & $379(51.8 \%)$ & 0.12 \\
\hline Diabetes mellitus & $52(25.1 \%)$ & $202(27.6 \%)$ & 0.47 \\
\hline Dyslipidemia & $93(44.9 \%)$ & $318(43.5 \%)$ & 0.72 \\
\hline $\mathrm{CHF}$ & $40(19.9 \%)$ & $122(17.6 \%)$ & 0.45 \\
\hline CKD & $30(14.5 \%)$ & $95(13.0 \%)$ & 0.58 \\
\hline Hemoglobin $[\mathrm{g} / \mathrm{dL}]$ & $11.0 \pm 1.8$ & $11.3 \pm 1.8$ & 0.05 \\
\hline Creatinine $[\mathrm{mg} / \mathrm{dL}]$ & $0.9 \pm 0.5$ & $0.9 \pm 0.6$ & 0.43 \\
\hline $\mathrm{HbA} 1 \mathrm{c}[\%]$ & $7.1 \pm 1.5$ & $6.9 \pm 1.5$ & 0.68 \\
\hline NT-proBNP [pg/dL] & $1107 \pm 2464$ & $1138 \pm 3610$ & 0.94 \\
\hline hsCRP [mg/dL] & $1.7 \pm 3.8$ & $1.4 \pm 2.6$ & 0.26 \\
\hline Post-operative hsCRP [mg/dL] & $11.8 \pm 6.2$ & $10.3 \pm 7.2$ & $0.04 *$ \\
\hline LA diameter $[\mathrm{mm}]$ & $42.4 \pm 8.4$ & $40.7 \pm 6.7$ & $0.02 *$ \\
\hline LA volume $[\mathrm{mL}]$ & $71.4 \pm 39.0$ & $63.0 \pm 33.3$ & $0.04 *$ \\
\hline$E / A$ & $1.08 \pm 0.53$ & $1.03 \pm 0.62$ & 0.36 \\
\hline$E / E^{\prime}$ & $16.9 \pm 11.8$ & $14.7 \pm 7.8$ & $0.04 *$ \\
\hline LVEF [\%] & $58.1 \pm 12.4$ & $58.3 \pm 12.2$ & 0.88 \\
\hline Type of surgery: & & & $<0.01 *$ \\
\hline CABG & $91(44.0 \%)$ & $408(55.8 \%)$ & \\
\hline Valve surgery & $72(34.8 \%)$ & $146(20.0 \%)$ & \\
\hline Aorta surgery & $19(9.2 \%)$ & $59(8.1 \%)$ & \\
\hline Combined $\mathrm{CABG}$ and valve surgery & $7(3.4 \%)$ & $21(2.9 \%)$ & \\
\hline Combined CABG and aorta surgery & $0(0 \%)$ & $2(0.3 \%)$ & \\
\hline Combined valve and aorta surgery & $11(5.3 \%)$ & $21(2.9 \%)$ & \\
\hline Miscellanous & $7(3.4 \%)$ & $74(10.1 \%)$ & \\
\hline Off-pump operation & $40(19.3 \%)$ & $236(32.3 \%)$ & $<0.01 \ddagger$ \\
\hline Mitral valve surgery & $44(21.3 \%)$ & $75(10.3 \%)$ & $<0.01 *$ \\
\hline Post-operative infection & $7(3.4 \%)$ & $27(3.7 \%)$ & 0.82 \\
\hline
\end{tabular}

${ }^{*} \mathrm{p}<0.05, \neq \mathrm{p}<0.01 ; \mathrm{CABG}$ — coronary artery bypass graft; CHF — congestive heart failure; CKD — chronic kidney disease; HbA1c — glycated hemoglobin; hsCRP — high sensitivity C-reactive protein; LA — left atrial; LVEF — left ventricular ejection fraction; NT-proBNP — N-terminal pro-B-type natriuretic peptide

Development of POAF occurred after mean $3.77 \pm 4.62$ post-operative days. Of the 207 patients with POAF, 41 patients were treated with anti-arrhythmic drugs, 18 patients underwent electrical cardioversion in the immediate postoperative period, 7 patients underwent scheduled electrical cardioversion after failure of converting to sinus rhythm with anti-arrhythmic drugs, and 141 patients received only rate-control drugs during hospitalization. A total of 6 patients remained in $\mathrm{AF}$ rhythm at discharge.

A multivariable logistic regression was performed for analysis of independent predictors of developing POAF. Older age showed borderline significance (OR 1.03, 95\% CI 0.99-1.06, $\mathrm{p}=0.06$ ) and concomitant mitral valvular surgery was an independent predictor for developing POAF (OR 3.73, 95\% CI 1.27-11.00, $\mathrm{p}=0.02$ ).

\section{Late occurrence of atrial fibrillation} during long-term follow up

Development of late AF occurred in 88 (9.4\%) patients during the mean follow up period of $78.1 \pm$ \pm 39.1 months. Baseline characteristics according to the development of late AF are compared in Table2. Patients with late AF were significantly older than those without $(61.6 \pm 12.0$ vs. $56.2 \pm 13.2$ year, $\mathrm{p}<0.01)$. Patients with occurrence of late 
Table 2. Patient characteristics according to development of late atrial fibrillation (AF).

\begin{tabular}{|c|c|c|c|}
\hline & Late $\mathrm{AF}(+) ; \mathbf{n}=\mathbf{8 8}$ & Late AF (-); $n=850$ & $\mathbf{P}$ \\
\hline Age [years] & $61.6 \pm 12.0$ & $56.2 \pm 13.2$ & $<0.01^{*}$ \\
\hline Male & $51(57.9 \%)$ & $499(58.7 \%)$ & 0.89 \\
\hline Hypertension & $46(52.3 \%)$ & $453(53.3 \%)$ & 0.86 \\
\hline Diabetes mellitus & $26(29.5 \%)$ & $228(26.8 \%)$ & 0.58 \\
\hline Dyslipidemia & $37(42.0 \%)$ & $374(44.0 \%)$ & 0.73 \\
\hline $\mathrm{CHF}$ & $22(25.9 \%)$ & $140(17.3 \%)$ & $0.05^{*}$ \\
\hline CKD & $19(21.6 \%)$ & $106(12.5 \%)$ & $0.02^{*}$ \\
\hline Hemoglobin $[\mathrm{g} / \mathrm{dL}]$ & $11.3 \pm 1.7$ & $11.3 \pm 1.8$ & 0.89 \\
\hline Creatinine $[\mathrm{mg} / \mathrm{dL}]$ & $1.0 \pm 0.4$ & $0.9 \pm 0.6$ & 0.38 \\
\hline $\mathrm{HbA1c}[\%]$ & $7.3 \pm 1.6$ & $6.9 \pm 1.4$ & 0.38 \\
\hline NT-proBNP [pg/dL] & $1093 \pm 1401$ & $1134 \pm 3490$ & 0.95 \\
\hline hsCRP [mg/dL] & $1.07 \pm 1.55$ & $1.47 \pm 3.00$ & 0.09 \\
\hline Post-operative hsCRP [mg/dL] & $9.9 \pm 5.4$ & $10.7 \pm 7.1$ & 0.47 \\
\hline LA diameter $[\mathrm{mm}]$ & $43.0 \pm 9.5$ & $40.9 \pm 6.9$ & 0.06 \\
\hline LA volume [mL] & $80.3 \pm 34.3$ & $63.8 \pm 34.7$ & $0.01 *$ \\
\hline$E / A$ & $1.08 \pm 0.56$ & $1.04 \pm 0.60$ & 0.57 \\
\hline $\mathrm{E} / \mathrm{e}^{\prime}$ & $21.1 \pm 15.3$ & $14.6 \pm 7.9$ & $<0.01 \mp$ \\
\hline LVEF (\%) & $56.2 \pm 12.6$ & $58.4 \pm 12.2$ & 0.10 \\
\hline Type of surgery: & & & $<0.01 \ddagger$ \\
\hline CABG & $35(39.8 \%)$ & $464(54.6 \%)$ & \\
\hline Valve surgery & $32(36.4 \%)$ & $186(21.9 \%)$ & \\
\hline Aorta surgery & $3(3.4 \%)$ & $75(8.8 \%)$ & \\
\hline Combined CABG and valve surgery & $5(5.7 \%)$ & $23(2.7 \%)$ & \\
\hline Combined CABG and aorta surgery & $0(0 \%)$ & $2(0.2 \%)$ & \\
\hline Combined valve and aorta surgery & $7(8.0 \%)$ & $25(2.9 \%)$ & \\
\hline Miscellanous & $6(6.8 \%)$ & $75(8.8 \%)$ & \\
\hline Off-pump operation & $25(28.4 \%)$ & $251(29.4 \%)$ & 0.83 \\
\hline Mitral valve surgery & $20(22.7 \%)$ & $99(11.6 \%)$ & $<0.01 \mp$ \\
\hline Post-operative infection & $1(1.1 \%)$ & $33(3.9 \%)$ & 0.15 \\
\hline Post-operative AF & $60(68.2 \%)$ & $147(17.3 \%)$ & $<0.01 \mp$ \\
\hline
\end{tabular}

${ }^{*} \mathrm{p}<0.05, \neq \mathrm{p}<0.01$; CABG — coronary artery bypass graft; CHF — congestive heart failure; CKD — chronic kidney disease; HbA1c glycated hemoglobin; hsCRP — high sensitivity C-reactive protein; LA — left atrial; LVEF — left ventricular ejection fraction; NT-proBNP — $\mathrm{N}$-terminal pro-B-type natriuretic peptide

$\mathrm{AF}$ showed higher incidence of congestive heart failure $(25.9 \%$ [22/88] vs. $17.3 \%$ [140/850], $\mathrm{p}=$ $=0.05)$ and chronic kidney disease $(21.6 \%$ [19/88] vs. $12.5 \%$ [106/850], $\mathrm{p}=0.02)$ than those without late AF. Proportion of patients with hypertension, diabetes, dyslipidemia, coronary artery disease and male sex was similar between the two groups. Pre-operative and post-operative hsCRP levels were not significantly different between the two groups. LA volume was significantly larger in patients with late AF than those without $(80.3 \pm 34.3$ vs. $63.8 \pm 34.7 \mathrm{~mL}$, $\mathrm{p}=0.01$ ) and septal $\mathrm{E} / \mathrm{e}^{\prime}$ ratio reflecting high $\mathrm{LV}$ end-diastolic pressure on the echocardiogram performed prior to surgery was significantly higher in patients with late AF $(21.1 \pm 15.3$ vs. $14.6 \pm 7.9, \mathrm{p}<0.01)$. A significantly higher proportion of patients with late AF underwent mitral valvular surgery $(22.7 \%$ [20/88] vs. $11.6 \%$ [99/850], p < 0.01).

Of patients who developed POAF, 147 (71.0\%) remained in sinus rhythm during long-term follow up, however, development of late AF occurred more frequently in patients with POAF than in those without (29.0\% [60/207] vs. 3.8\% [28/731], $\mathrm{p}<0.01$ ) (Fig. 1). 


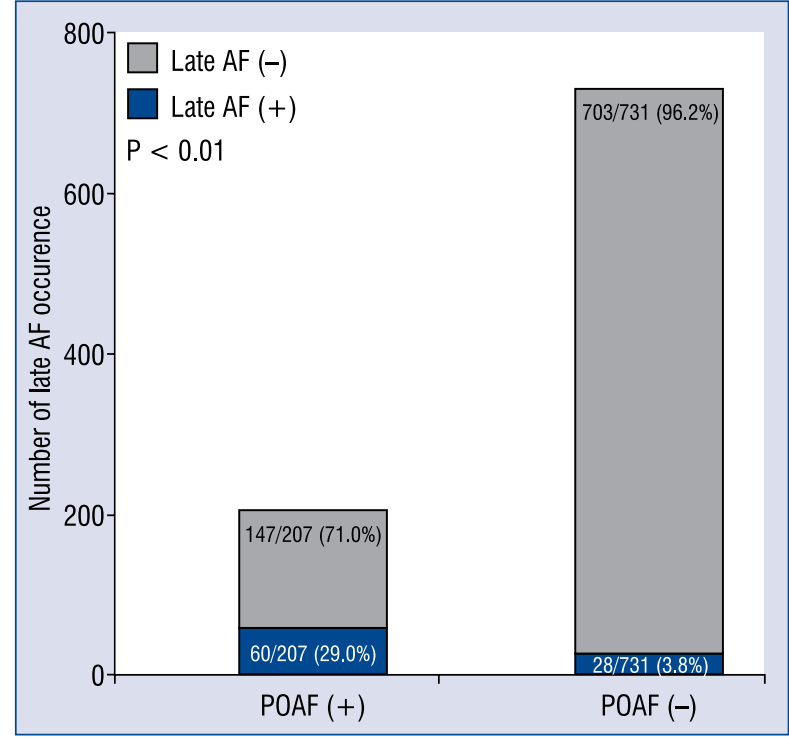

Figure 1. Development of late atrial fibrillation (AF) occurred more frequently in patients with post-operative atrial fibrillation (POAF) than in those without POAF [29.0\% (60/207) vs. $3.8 \%(28 / 731), p<0.01]$.

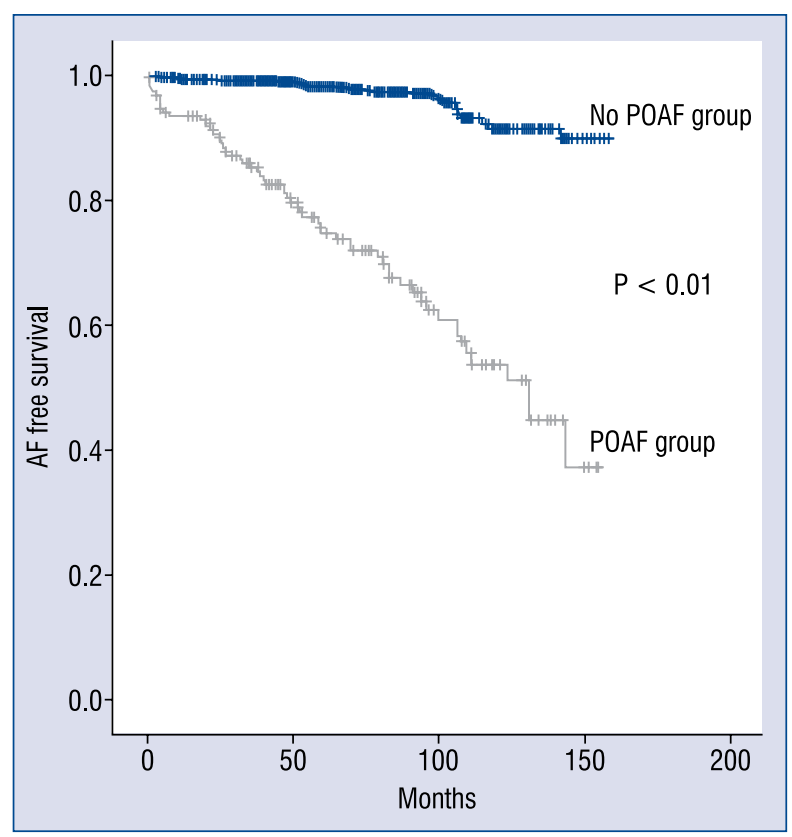

Figure 2. The Kaplan-Meier curve of atrial fibrillation (AF) free survival according to development of postoperative atrial fibrillation (POAF) during long-term follow up. The POAF group showed a significantly lower cumulative AF free survival than the no POAF group $(p<0.01)$.
Atrial fibrillation free survival and predictors of late occurrence of atrial fibrillation

Figure 2 shows AF free survival according to development of POAF during long-term follow up. The POAF group showed a significantly lower cumulative AF free survival than the no POAF group $(\mathrm{p}<0.01)$ and the POAF group curve demonstrates a steeper curve and continuous divergence of gradient suggesting ongoing risk of $\mathrm{AF}$ apart from traditional risk factors during long-term follow up.

A multivariable logistic regression was performed for analysis of independent predictors for developing late AF. In multivariate analysis, E/e' ratio (HR 1.04, 95\% CI 1.00-1.08, p = 0.04) was an independent predictor of late occurrence of AF and an episode of POAF (HR 27.12, 95\% CI 8.46-86.96, $\mathrm{p}<0.01)$ was the most powerful predictor (Table 3 ).

\section{Discussion}

This study demonstrates that POAF is significantly associated with an increased risk of late $\mathrm{AF}$ occurrence and POAF gives ongoing risk of developing late $\mathrm{AF}$ during long-term follow up. In addition, E/e' ratio reflecting high LV end-diastolic pressure at the time of surgery was an independent predictor for developing late AF. Concomitant mitral valvular surgery caused independent risk for developing POAF, however, it was not a risk factor for late $\mathrm{AF}$.

Although short-term complications and outcomes of POAF are well known, late recurrence rate of $\mathrm{AF}$ during long-term follow up in patients with POAF receiving OHS has not yet been established. Recent studies have suggested that POAF following CABG carries a risk of future $\mathrm{AF}$ recurrence compared to patients without an episode of POAF $[5,6]$. One study reported that $25.4 \%$ of POAF patients with no history of AF who underwent primary coronary bypass surgery had late AF compared with $3.6 \%$ of patients with no POAF at median follow-up of 6 years [12]. In another study, a higher incidence of late AF occurrence was observed in the POAF group than in the no POAF group $(18.9 \%$ vs. $2.2 \%, \mathrm{p}<0.001)$ during the follow up period of $41 \pm 23$ months [6]. Results of our study were consistent with those of previous studies reporting that POAF carries higher risk for late recurrence of AF. In multivariate analysis, it was found that $29.0 \%$ of POAF patients 
Table 3. Predictors for occurrence of late atrial fibrillation (AF) during long-term follow up; multivariable analysis.

\begin{tabular}{|c|c|c|c|c|}
\hline \multirow{2}{*}{$\begin{array}{l}\text { "No late } A F^{\prime \prime} \text { as } \\
\text { a reference } \\
\text { population }\end{array}$} & \multicolumn{2}{|c|}{ Univariate analysis } & \multicolumn{2}{|c|}{ Multivariate analysis } \\
\hline & Hazard ratio $(95 \% \mathrm{CI})$ & $\mathbf{P}$ & Hazard ratio $(95 \% \mathrm{Cl})$ & $\mathbf{P}$ \\
\hline Age & $1.04(1.02-1.06)$ & $<0.01 \ddagger$ & 1.02 (0.97-1.07) & 0.56 \\
\hline $\mathrm{CHF}$ & $1.67(0.99-2.81)$ & 0.05 & $0.71(0.21-2.45)$ & 0.59 \\
\hline CKD & $1.93(1.12-3.34)$ & $0.02 *$ & $2.54(0.75-8.60)$ & 0.14 \\
\hline LA volume & $1.01(1.00-1.02)$ & $0.03 *$ & 1.01 (0.99-1.08) & 0.08 \\
\hline$E / e^{\prime}$ & $1.05(1.03-1.08)$ & $<0.01 \ddagger$ & $1.04(1.00-1.08)$ & $0.04^{*}$ \\
\hline Mitral valve surgery & $2.23(1.30-3.83)$ & $<0.01 \ddagger$ & $0.53(0.14-1.95)$ & 0.34 \\
\hline Post-operative AF & $10.25(6.33-16.60)$ & $<0.01 \ddagger$ & $27.12(8.46-86.96)$ & $<0.01 \mp$ \\
\hline
\end{tabular}

${ }^{*} \mathrm{p}<0.05, \neq \mathrm{p}<0.01 ; \mathrm{Cl}$ - confidence interval; CHF — congestive heart failure; CKD — chronic kidney disease; LA — left atrial

developed any type of AF during long-term follow up, with an adjusted risk ratio of 27.1 for late AF compared with no POAF patients. Interestingly, this relative risk persisted during long-term follow up. This study is unique in the following points: first, despite previous studies which confined their analysis to patients undergoing isolated CABG surgery, this is the first study evaluating long-term consequences of POAF among patients undergoing general OHS. In comparing the risk of POAF and late $\mathrm{AF}$ according to the type of surgery, it was found that more complex procedures such as combined surgery with valvular operation caused significantly higher rates of POAF rather than isolated CABG. However, the type of surgery was not an independent predictor of late $\mathrm{AF}$ occurrence. This can be explained that type and complexity of surgery may influence the degree of inflammation and alteration of hemodynamic status which can induce AF during post-operative period, however, type of surgery rarely affects development of AF after recovery of post-operative condition and correction of pre-operative disease. Similarly, OPCAB did not affect the incidence of POAF as previously reported [13] and we found that the development of late AF was not related with OPCAB. Second, among the research to date, the present study evaluated the occurrence of late $\mathrm{AF}$ for the longest follow up period (mean follow up period of $78.1 \pm$ 39.1 months).

Diastolic dysfunction is associated with progressive LA enlargement and stretching of pulmonary veins due to LA pressure overload which may cause arrhythmogenic activity [14]. The association between $\mathrm{AF}$ and diastolic dysfunction has been previously reported and diastolic dysfunc- tion was a predictor of new onset $\mathrm{AF}[15,16]$. The septal E/e' ratio, a diastolic parameter, is an easy and non-invasive echocardiographic marker of LV filling pressure. The septal $\mathrm{E} / \mathrm{e}^{\prime}$ ratio $\geq 11$ is an index of elevated LV filling pressure ( $>15 \mathrm{~mm} \mathrm{Hg}$ ) with a sensitivity of $75 \%$ and a specificity of $93 \%$ and reflects higher LA pressure [17]. Elevated $\mathrm{E} / \mathrm{e}$ ' ratio served as a predictor of recurrence after catheter ablation of AF [18] and an independent predictor of AF recurrence after cardioversion [19]. Septal E/e' ratio was also an independent predictor of mortality in patients with non-valvular AF [20].

Patients with either POAF or late AF were significantly older than those without POAF or late AF. Advanced age is the most consistent predictor for the development of POAF [21]. Age related degenerative and inflammatory modifications in atrial anatomy and altered electrophysiological properties are preconditions for POAF with acute surgical trauma and inflammation likely providing the inciting factors for POAF $[22,23]$. However, advanced age was not an independent predictor for developing late AF in this study, suggesting ongoing risk of $\mathrm{AF}$ in $\mathrm{POAF}$ patients apart from traditional risk factors during long-term follow up.

The findings of this study suggest that POAF is not a benign condition and that they can lead to a high incidence of future $\mathrm{AF}$ during long-term follow up, and POAF can be regarded as a risk marker for development of late AF. A previous study reported an association of POAF with decreased long-term survival independent of co-existing cardiovascular disease and comorbidities [24] and the presented results provide evidence supporting low probability of long-term survival in patients with POAF. POAF was the most powerful predictor of late occurrence 
of $\mathrm{AF}$ and septal $\mathrm{E} / \mathrm{e}$ ' ratio was found to be another predictor of late $\mathrm{AF}$ occurrence.

A previously higher SYNTAX score [25] and HATCH score [26] were independent predictors for developing POAF after CABG. Vitamin D deficiency was evaluated to predict the development of POAF in patients receiving CABG, which showed insignificant factor [27]. Whether these parameters have predictive value in the development of late $\mathrm{AF}$ were not evaluated yet and further studies are necessary. Although several pharmacologic or non-pharmacologic interventions have been shown to reduce the incidence of POAF [21], whether or not these interventions translate to a significant reduction of peri-operative complications and further reduce the incidence of late AF is still controversial. Future studies are required about strategy for prevention of late $\mathrm{AF}$ development in patients with POAF.

\section{Limitations of the study}

There are several limitations in the present study. This is a retrospective investigation and asymptomatic AF occurrence might have been missed in some patients during follow up. Twelvelead surface ECG and 24-h or 48-h Holter monitoring might have limitations in patients with paroxysmal AF episodes. Serial evaluation of hsCRP concentration was not performed, therefore, the possibility of a relationship between CRP level and POAF cannot be fully excluded [28]. Because of heterogeneity of operation type, detailed analysis of peri-operative data was difficult. Excluding patients with insufficient follow up can be regarded as a selection bias.

\section{Conclusions}

Post-operative atrial fibrillation is significantly associated with an increased risk of late $\mathrm{AF}$ recurrence during long-term follow up. Careful concern regarding late recurrence of $\mathrm{AF}$ with serial evaluation of rhythm status is required in patients with peri-operative atrial fibrillation.

\section{Conflict of interest: None declared}

\section{References}

1. JCS Joint Working Group. Guidelines for Pharmacotherapy of Atrial Fibrillation (JCS 2013). Circ J. 2014; 78(8): 1997-2021, indexed in Pubmed: 24965079.

2. Aranki SF, Shaw DP, Adams DH, et al. Predictors of atrial fibrillation after coronary artery surgery. Current trends and impact on hospital resources. Circulation. 1996; 94(3): 390-397, indexed in Pubmed: 8759081.
3. Creswell LL, Schuessler RB, Rosenbloom M, et al. Hazards of postoperative atrial arrhythmias. Ann Thorac Surg. 1993; 56(3): 539-549, indexed in Pubmed: 8379728.

4. Mathew JP, Parks R, Savino JS, et al. Atrial fibrillation following coronary artery bypass graft surgery: predictors, outcomes, and resource utilization. MultiCenter Study of Perioperative Ischemia Research Group. JAMA. 1996; 276(4): 300-306, indexed in Pubmed: 8656542.

5. Antonelli D, Peres D, Freedberg NA, et al. Incidence of postdischarge symptomatic paroxysmal atrial fibrillation in patients who underwent coronary artery bypass graft: long-term follow-up. Pacing Clin Electrophysiol. 2004; 27(3): 365-367, indexed in Pubmed: 15009865.

6. Lee SH, Kang DR, Uhm JS, et al. New-onset atrial fibrillation predicts long-term newly developed atrial fibrillation after coronary artery bypass graft. Am Heart J. 2014; 167(4): 593-600.e1, doi: 10.1016/j.ahj.2013.12.010, indexed in Pubmed: 24655710.

7. Expert Panel on Detection, Evaluation, and Treatment of High Blood Cholesterol in Adults. Executive Summary of The Third Report of The National Cholesterol Education Program (NCEP) Expert Panel on Detection, Evaluation, And Treatment of High Blood Cholesterol In Adults (Adult Treatment Panel III). JAMA. 2001; 285(19): 2486-2497, indexed in Pubmed: 11368702.

8. Elahi M, Hadjinikolaou L, Galiñanes M. Incidence and clinical consequences of atrial fibrillation within 1 year of first-time isolated coronary bypass surgery. Circulation. 2003; 108 Suppl 1: II207-II212, doi: 10.1161/01.cir.0000089188.45285.fd, indexed in Pubmed: 12970234.

9. Sahn DJ, DeMaria A, Kisslo J, et al. Recommendations regarding quantitation in M-mode echocardiography: results of a survey of echocardiographic measurements. Circulation. 1978; 58(6): 1072-1083, indexed in Pubmed: 709763.

10. Lang RM, Bierig M, Devereux RB, et al. Recommendations for chamber quantification: a report from the American Society of Echocardiography's Guidelines and Standards Committee and the Chamber Quantification Writing Group, developed in conjunction with the European Association of Echocardiography, a branch of the European Society of Cardiology. J Am Soc Echocardiogr. 2005; 18(12): 1440-1463, doi: 10.1016/j. echo.2005.10.005, indexed in Pubmed: 16376782.

11. Lee JK, Klein GJ, Krahn AD, et al. Rate-control versus conversion strategy in postoperative atrial fibrillation: a prospective, randomized pilot study. Am Heart J. 2000; 140(6): 871-877, doi: 10.1067/mhj.2000.111104, indexed in Pubmed: 11099990.

12. Ahlsson A, Fengsrud E, Bodin L, et al. Postoperative atrial fibrillation in patients undergoing aortocoronary bypass surgery carries an eightfold risk of future atrial fibrillation and a doubled cardiovascular mortality. Eur J Cardiothorac Surg. 2010; 37(6): 1353-1359, doi: 10.1016/j.ejcts.2009.12.033, indexed in Pubmed: 20138531.

13. Lewicki Ł, Siebert J, Rogowski J. Atrial fibrillation following off-pump versus on-pump coronary artery bypass grafting: Incidence and risk factors. Cardiol J. 2016 [Epub ahead of print]; 23(5): 518-523, doi: 10.5603/CJ.a2016.0066, indexed in Pubmed: 27665857.

14. Kalifa J, Jalife J, Zaitsev AV, et al. Intra-atrial pressure increases rate and organization of waves emanating from the superior pulmonary veins during atrial fibrillation. Circulation. 2003; 108(6): 668-671, doi: 10.1161/01.CIR.0000086979.39843.7B, indexed in Pubmed: 12900337.

15. Jaïs P, Peng JT, Shah DC, et al. Left ventricular diastolic dysfunction in patients with so-called lone atrial fibrillation. J Car- 
diovasc Electrophysiol. 2000; 11(6): 623-625, indexed in Pubmed: 10868734.

16. Tsang TSM, Gersh BJ, Appleton CP, et al. Left ventricular diastolic dysfunction as a predictor of the first diagnosed nonvalvular atrial fibrillation in 840 elderly men and women. J Am Coll Cardiol. 2002; 40(9): 1636-1644, indexed in Pubmed: 12427417.

17. Sohn DW, Song JM, Zo JH, et al. Mitral annulus velocity in the evaluation of left ventricular diastolic function in atrial fibrillation. J Am Soc Echocardiogr. 1999; 12(11): 927-931, indexed in Pubmed: 10552353.

18. Hirai T, Cotseones G, Makki N, et al. Usefulness of left ventricular diastolic function to predict recurrence of atrial fibrillation in patients with preserved left ventricular systolic function. Am J Cardiol. 2014; 114(1): 65-69, doi: 10.1016/j.amjcard.2014.03.061, indexed in Pubmed: 24819904.

19. Fornengo C, Antolini M, Frea S, et al. Prediction of atrial fibrillation recurrence after cardioversion in patients with left-atrial dilation. Eur Heart J Cardiovasc Imaging. 2015; 16(3): 335-341, doi: 10.1093/ehjci/jeu193, indexed in Pubmed: 25274966.

20. Okura H, Takada Y, Kubo T, et al. Tissue Doppler-derived index of left ventricular filling pressure, E/E', predicts survival of patients with non-valvular atrial fibrillation. Heart. 2006; 92(9): 1248-1252, doi: 10.1136/hrt.2005.082594, indexed in Pubmed: 16449507.

21. Echahidi N, Pibarot P, O’Hara G, et al. Mechanisms, prevention, and treatment of atrial fibrillation after cardiac surgery. J Am Coll Cardiol. 2008; 51(8): 793-801, doi: 10.1016/j.jacc.2007.10.043, indexed in Pubmed: 18294562.
22. Greenberg JW, Lancaster TS, Schuessler RB, et al. Postoperative atrial fibrillation following cardiac surgery: a persistent complication. Eur J Cardiothorac Surg. 2017 [Epub ahead of print], doi: 10.1093/ejcts/ezx039, indexed in Pubmed: 28369234.

23. Allessie MA, Boyden PA, Camm AJ, et al. Pathophysiology and prevention of atrial fibrillation. Circulation. 2001; 103(5): 769777, indexed in Pubmed: 11156892.

24. Villareal RP, Hariharan R, Liu BC, et al. Postoperative atrial fibrillation and mortality after coronary artery bypass surgery. J Am Coll Cardiol. 2004; 43(5): 742-748, doi: 10.1016/j. jacc.2003.11.023, indexed in Pubmed: 14998610.

25. Cerit L, Duygu H, Gulsen K, et al. Is SYNTAX Score Predictive of Atrial Fibrillation after On-Pump Coronary Artery Bypass Graft Surgery? Korean Circ J. 2016; 46(6): 798-803, doi: 10.4070/ kcj.2016.46.6.798, indexed in Pubmed: 27826338.

26. Emren V, Aldemir M, Duygu H, et al. Usefulness of HATCH score as a predictor of atrial fibrillation after coronary artery bypass graft. Kardiol Pol. 2016 [Epub ahead of print]; 74(8): 749-753, doi: 10.5603/KP.a2016.0045, indexed in Pubmed: 27040011.

27. Cerit L, Kemal H, Gulsen K, et al. Relationship between Vitamin $\mathrm{D}$ and the development of atrial fibrillation after on-pump coronary artery bypass graft surgery. Cardiovasc J Afr. 2017; 28(2): 104-107, doi: 10.5830/CVJA-2016-064, indexed in Pubmed: 27701486 .

28. Harada M, Van Wagoner DR, Nattel S. Role of inflammation in atrial fibrillation pathophysiology and management. Circ J. 2015; 79(3): 495-502, doi: 10.1253/circj.CJ-15-0138, indexed in Pubmed: 25746525. 